\title{
Applications of VANETs: Present \& Future
}

\author{
Vishal Kumar $^{1}$, Shailendra Mishra ${ }^{1}$, Narottam Chand ${ }^{2}$ \\ ${ }^{1}$ Department of Computer Science \& Engineering, Bipin Tripathi Kumaon Institute of Technology, Dwarahat, India \\ ${ }^{2}$ Department of Computer Science \& Engineering, National Institute of Technology, Hamirpur, India \\ Email: vishalkumar@ieee.org, smishra@kecua.ac.in, nar@nith.ac.in
}

Received 2012

\begin{abstract}
The Vehicular Safety Consortium (VSC), the Crash-Avoidance Metrics Partnership (CAMP) consortium and the Vehicle Infrastructure Initiative (VII) [1] along with the giants of the light-duty vehicle manufactures, are working to develop pre-competitive safety technologies and various applications that can be offered in Vehicular ad-hoc Networks (VANETs), a special kind mobile ad-hoc networks where wireless equipped element called on-board unit (OBU) in vehicles form a network with the Roadside unit (RSU) without any additional infrastructure. In this paper, we are primarily categorizing various possible applications of vehicular network, along with its features, and implementations in the real world.
\end{abstract}

Keywords: Roadside Unit; WAVE; VSC; CAMP; VII

\section{Introduction}

As the vehicles are growing, the possibility of accidents has also increased. According to the National Highway Traffic Safety Administration (NHTSA) [1,4], there are around 43000 deaths /year, 2.7 million people injured/ year and $\$ 230$ billion social cost. It is required to make our vehicles a bit intelligent so that we can reduce the possibilities of accidents. A vehicular ad-hoc network (VANET) adds ability in the vehicles. Dedicated Short Range Communications (DSRC) and IEEE 802.11p Wireless Access for Vehicular Environment (WAVE) [2, 3] have been approved as standards for PHY and MAC layers for vehicular networks. The IEEE 802.11p WAVE standardization process originates from the allocation of the DSRC spectrum band of $5.9 \mathrm{GHz}$ with a bandwidth of $75 \mathrm{MHz}$ and approximate range of $1000 \mathrm{~m}$. The main aim of DSRC and the IEEE 802.11p WAVE standard is to define rules for low connection setup delay, fast network recognition and the differentiation of applications for normal and emergency use. They allow a high throughput communication with low delay among vehicles. This leads to efficient emergency communications. For example, in the case of accidents, an alerting message transmitted among vehicles can be faster and, thus, well-timed, rather than communications sent through an infrastructure network (such as cellular systems).

\section{Characteristics of Vanets}

Many different and sometimes competing design goals have to be taken into account for VANETs to ensure their commercial success. When equipped with WAVE (Wireless Access for Vehicular Environment, a novel type of wireless access dedicated to vehicle-to-vehicle and vehicle-to-roadside communications), in Figure 1 it forms a highly dynamic network.

Although, some characteristics of VANETs resembles with the characteristics of MANETs [6, 8] but there are specific features which can be categorized as follows:

1) Highly dynamic topology

The high speed of the vehicles along with the availability of choices of multiple paths defines the dynamic topology of VANETs.

2) Frequent disconnected network

The high speed of the vehicles in one way defines the dynamic topology whereas on the other hand necessitates the frequent requirements of the roadside unit lack of which results a frequent disconnections.

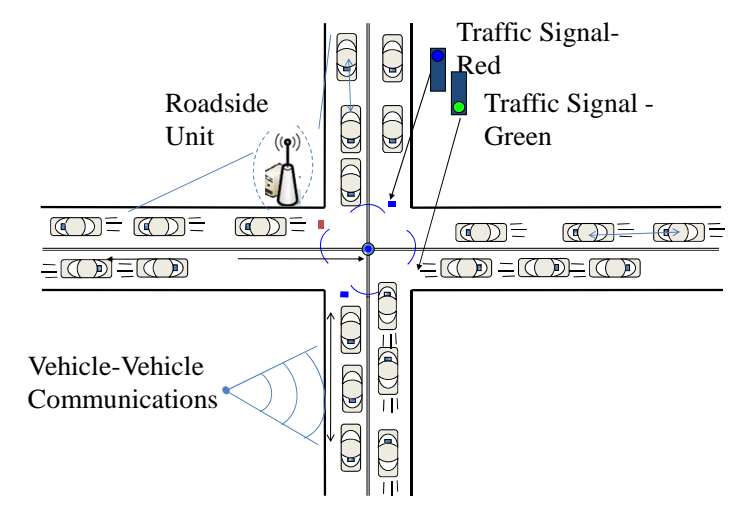

Figure 1. Vehicular Ad Hoc Networks. 


\section{3) Mobility modeling and Prediction}

The prediction of vehicle position and their movements is very difficult. This features of mobility modeling and prediction in VANETs is based on the availability of predefined roadmaps models. The speed of the vehicles is again an important for efficient network design.

\section{4) Communication Environment}

Once we are having a mobility model, yet we are not done. As the mobility model may have different features depending upon road architecture, highways, or city environments. Communicating in these situations has to be taken care.

\section{5) Hard delay constraints}

At the time of emergency, delivery of messages on time is a critical problem. Therefore, handle such situations rather talking only about high data rates in not sufficient.

6) Interaction with onboard sensors

Sensors are the mode of communications. Sensors can read data related to velocity of the vehicle, direction and can communicate to the data center. Thus sensors can be used in link formation and in routing protocols.

7) Unlimited Battery Power and Storage

Nodes in VANETs do not suffer power and storage limitation as in sensor networks; therefore optimizing duty cycle is not as relevant as in sensor networks.

\section{Applications of Vanets}

The RSU can be treated as an access point or router or even a buffer point which can store data and provide data when needed [5]. All data on the RSUs are uploaded or downloaded by vehicles. A classification of applications is also done by [20] as Car to Car Traffic applications, Car to Infrastructure applications, Car to Home applications and Routing based applications. The authors in [19] discusses about the various attacks based on their classification. Based on the type of communication either V2I or $\mathrm{V} 2 \mathrm{~V}$, we are arranging the applications of VANETs into following classes:

1) Safety oriented,

2) Commercial oriented

3) Convenience oriented and

4) Productive Applications

\subsection{Safety Applications}

Safety applications include monitoring of the surrounding road, approaching vehicles, surface of the road, road curves etc. The Road safety applications can be classified as:

1) Real-time traffic: The real time traffic data can be stored at the RSU and can be available to the vehicles whenever and wherever needed. This can play an impor- tant role in solving the problems such as traffic jams, avoid congestions and in emergency alerts such as accidents etc.

2) Co-operative Message Transfer: Slow/Stopped Vehicle will exchange messages and co-operate to help other vehicles. Though reliability and latency would be of major concern, it may automate things like emergency braking to avoid potential accidents. Similarly, emergency electronic brake-light may be another application.

3) Post Crash Notification: A vehicle involved in an accident would broadcast warning messages about its position to trailing vehicles so that it can take decision with time in hand as well as to the highway patrol for tow away support as depicted in Figure 2.

4) Road Hazard Control Notification: Cars notifying other cars about road having landslide or information regarding road feature notification due to road curve, sudden downhill etc.

5) Cooperative Collision Warning: Alerts two drivers potentially under crash route so that they can mend their ways [7].

6) Traffic Vigilance: The cameras can be installed at the RSU that can work as input and act as the latest tool in low or zero tolerance campaign against driving offenses [9].

\subsection{Commercial Applications}

Commercial applications will provide the driver with the entertainment and services as web access, streaming audio and video. The Commercial applications can be classified as:

1) Remote Vehicle Personalization/ Diagnostics: It helps in downloading of personalized vehicle settings or uploading of vehicle diagnostics from/to infrastructure.

2) Internet Access: Vehicles can access internet through RSU if RSU is working as a router.

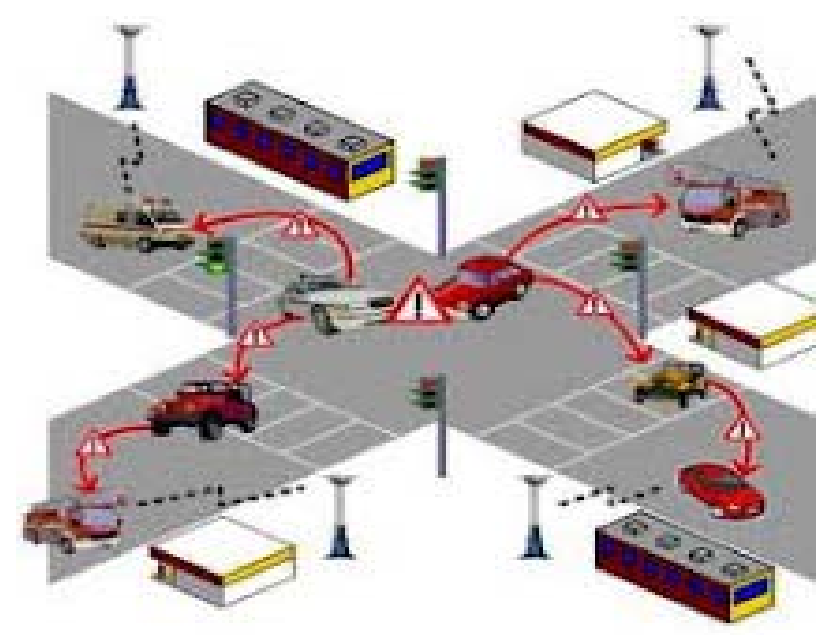

Figure 2. Emergency situation Notification. 
3) Digital map downloading: Map of regions can be downloaded by the drivers as per the requirement before traveling to a new area for travel guidance. Also, Content Map Database Download acts as a portal for getting valuable information from mobile hot spots or home stations.

4) Real Time Video Relay: On-demand movie experience will not be confined to the constraints of the home and the driver can ask for real time video relay of his favorite movies.

5) Value-added advertisement: This is especially for the service providers, who want to attract customers to their stores. Announcements like petrol pumps, highways restaurants to announce their services to the drivers within communication range. This application can be available even in the absence of the Internet.

\subsection{Convenience Applications}

Convenience application mainly deals in traffic management with a goal to enhance traffic efficiency by boosting the degree of convenience for drivers. The Convenience applications can be classified as:

1) Route Diversions: Route and trip planning can be made in case of road congestions.

2) Electronic Toll Collection: Payment of the toll can be done electronically through a Toll Collection Point as shown in Figure 3. A Toll collection Point shall be able to read the OBU of the vehicle. OBUs work via GPS [17] and the on-board odometer or techograph as a back-up to determine how far the Lorries have travelled by reference to a digital map and GSM to authorize the payment of the toll via a wireless link. TOLL application is beneficial not only to drivers but also to toll operators.

3) Parking Availability: Notifications regarding the availability of parking in the metropolitan cities helps to find the availability of slots in parking lots in a certain geographical area.

4) Active Prediction: It anticipates the upcoming topography of the road, which is expected to optimize fuel usage by adjusting the cruising speed before starting a descent or an ascent. Secondly, the driver is also assisted [10].

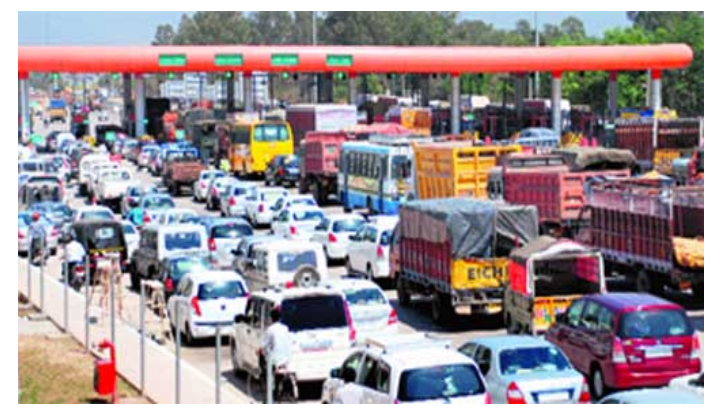

Figure 3. Electronic Toll Collection in India.

\subsection{Productive Applications}

We are intentionally calling it productive as this application is additional with the above mentioned applications. The Productive applications can be classified as:

1) Environmental Benefits: AERIS research program [11] is to generate and acquire environmentally-relevant real-time transportation data, and use these data to create actionable information that support and facilitate "green" transportation choices by transportation system users and operators. Employing a multi-modal approach, the AERIS program will work in partnership with the vehicle-to-vehicle (V2V) communications research effort to better define how connected vehicle data and applications might contribute to mitigating some of the negative environmental impacts of surface transportation.

2) Time Utilization: If a traveler downloads his email, he can transform jam traffic into a productive task and read on-board system and read it himself if traffic stuck. One can browse the Internet when someone is waiting in car for a relative or friend.

3) Fuel Saving: When the TOLL system application for vehicle collects toll at the toll booths without stopping the vehicles, the fuel around $3 \%$ is saved, which is consumed when a vehicles as an average waits normally for 2-5 minutes.

\section{Future}

As mobiles are familiar and used by us in our day to day life, similarly the future of VANETs is undoubtedly secure. It has become the part of the government projects. In India, National Highways Authority of India (NHAI) [12] is planning to replace manual toll collections at plazas with electronic toll collection (ETC) systems across the country. The ETC system will be based on radio frequency identification (RFID), which will be complemented by a wireless on-board unit (OBU) on a vehicle, as well as a stationery roadside unit (RSU) at the toll plaza.

Australian police in New South Wales (NSW) and Victoria are considering the introduction of a new type of laser speed camera, which can catch drivers using mobile phones, as well as speeding motorists from half a mile away [13]. The cameras, known as Concept II, have been manufactured by Tele-Traffic UK and are already in use by UK's Dorset police as the latest tool in their zero tolerance campaign against driving offenses. Similarly, various projects are running in various countries to employ VANETs in traffic safety and efficiency.

There are many other challenges left that will have a strong influence on the future of VANETs. Although, to show the impact of VANETs on traffic safety, and efficiency, a number of simulators are available namely EstiNet, ns-2, TRANS [14-16] and many more, but study of 
driver's behavior and reaction [18] when additional information is provided through VANETs is also a challenge. The adoption of VANETs in the market is another challenge as there are many players in the game.

\section{Conclusions}

In this paper, we first gave a description of architecture, standards \& protocols of vehicular ad hoc networks, followed by the characteristics described in Section II. Section III describes various applications based on its classification. Section IV is giving the implementation of applications at present and in future in countries that are deploying VANETs in one way or the other. Although the works are numerous, there are still issues which may be untouched. However, we want to clarify that the list of applications identified here is not exhaustive. We hope, at least, the study in this paper would contribute in new research directions.

\section{REFERENCES}

[1] Vehicle Safety Communications Project, Final Report, DOT HS 810 591, April 2006.

[2] IEEE, "Draft Amendment to STANDARD FOR Information technology-Telecommunications and information exchange between systems-LAN/MAN Specific Requirements-Part 11: Wireless LAN Medium Access Control (MAC) and Physical Layer (PHY) specifications: Wireless Access in Vehicular Environments (WAVE)”.

[3] “IEEE 802.11p Task Group,” http://grouper.ieee.org/groups/scc32/dsrc/index.html.

[4] www.nhtsa.gov

[5] V. Kumar and N. Chand, "Efficient Data Scheduling in VANETs," in Journal of Computing, Vol.-2, No.-8, September 2010, pp. 32-37.
[6] K. C. Lee, U. Lee and M. Gerla, "Survey of Routing Protocols in Vehicular Ad Hoc Networks," in Advances in Vehicular Ad-Hoc Networks: Developments and Challenges, IGI Global, Oct, 2009.

[7] X. Yang, L. Liu and N. Vaidya, "A vehicle-to-vehicle communication protocol for cooperative collision warning," $1^{\text {st }}$ Annual International conference on Mobile and Ubiquitous Systems: Networking \& Services, MOBIQUITOUS’04, pp. 114-123.

[8] V. Kumar, "Priority Based Data Scheduling in VANETs,” M. Tech Thesis, NIT Hamirpur, 2010.

[9] http://www.teletrafficuk.com/products-concept-ii.htm

[10] www.scania.com

[11] www.its.dot.gov/aeris

[12] http://www.nhai.org/

[13] www.roadtraffic-technology.com

[14] www.estinet.com

[15] www.isi.edu/nsnam/ns

[16] www.lca.epfl.ch/projects/trans

[17] R. B. Thompson, “Global Positioning System (GPS): The Mathematics of Satellite Navigation,” MathCAD library, http://www.mathsoft.com/appsindex.html. 1998.

[18] A. Smiley, M. Vernet, G. Labiale and A. Pavzie, "Navigation and Guidance Displays Impact on Driver Performance: Toward An Intelligent Transport System," Proceedings of the First World Congress on Applications of Transport Telematics and Intelligent vehicle Highway Systems, Vol. 4, 1994, pp. 1852-1859. http://pcquest.ciol.com/content/technology/2009/1090201 01.asp

[19] A. Aijaz, B. Bochow, F. D“otzer, A. Festag, M. Gerlach, R. Kroh and T. Leinm"uller, "Attacks on Inter Vehicle Communication Systems - an Analysis," The Network on Wheels Project, Tech. Rep., 2005. Available: http://www.network-on-wheels.de/documents.html 